\title{
Optical image processing with metasurface dark modes
}

\author{
Ann Roberts ${ }^{1, *}$, Daniel E. Gómez ${ }^{2}$, And Timothy J. Davis ${ }^{1}$ \\ ${ }^{1}$ School of Physics, The University of Melbourne, Victoria 3010, Australia \\ ${ }^{2}$ School of Applied Chemistry and Environmental Science, RMIT University, GPO Box 2476, Melbourne Victoria 3001, Australia \\ *Corresponding author: ann.roberts@unimelb.edu.au
}

Compiled July 26, 2018

\begin{abstract}
Here we consider image processing using optical modes of metasurfaces with an angle-dependent excitation. These spatially dispersive modes can be used to directly manipulate the spatial frequency content of an incident field suggesting their use as ultra-compact alternatives for analog optical information processing. A general framework for describing the filtering process in terms of the optical transfer functions is provided. In the case where the relevant mode cannot be excited with a normally incident plane wave (a dark mode) high pass filtering is obtained. We provide examples demonstrating filtering of both amplitude and pure phase objects.
\end{abstract}

(C) 2018 Optical Society of America

OCIS codes: (100.0100) Image processing; (050.6624) Subwavelength structures; (160.3918) Metamaterials; (110.4850) Optical transfer functions; (070.6110) Spatial filtering.

http://dx.doi.org/10.1364/ao.XX.XXXXXX

\section{INTRODUCTION}

Metasurfaces consisting of regular arrays of subwavelength resonators are attracting increasing interest as alternative, compact means to manipulate light in devices including lenses, holograms and polarizers [1, 2]. There is also an emerging realization that metasurfaces and metamaterials may play a role in optical information processing to perform mathematical operations on wavefields [3-8]. The field of optical information processing is well-evolved, with the classic configuration being a $4-f$ system consisting of two lenses and a spatial filter that can alter the phase or amplitude of the transmitted field (Fig. 1(a)) [9]. Although this configuration is flexible and extremely powerful, the requirement for lenses, and sufficient propagation length, leads to systems being bulky and heavy. Photonic crystals have been demonstrated as alternatives to conventional spatial filtering for beam cleaning applications [10] and designs for performing spatial differentiation have been proposed [11]. By tuning the characteristic geometry of photonic crystals, modulation of the spatial frequency content of transmitted or reflected optical fields can be achieved. Other methods proposed for performing direct spatial-frequency filtering have included phase-shifted Bragg gratings [12-14], thin-film slab waveguides [15-17] and other multilayer thin film stacks [18-20]. An analogue computing approach based on Brewster reflection [21] has been proposed and most recently, the use of thin metallic films in the Kretschmann

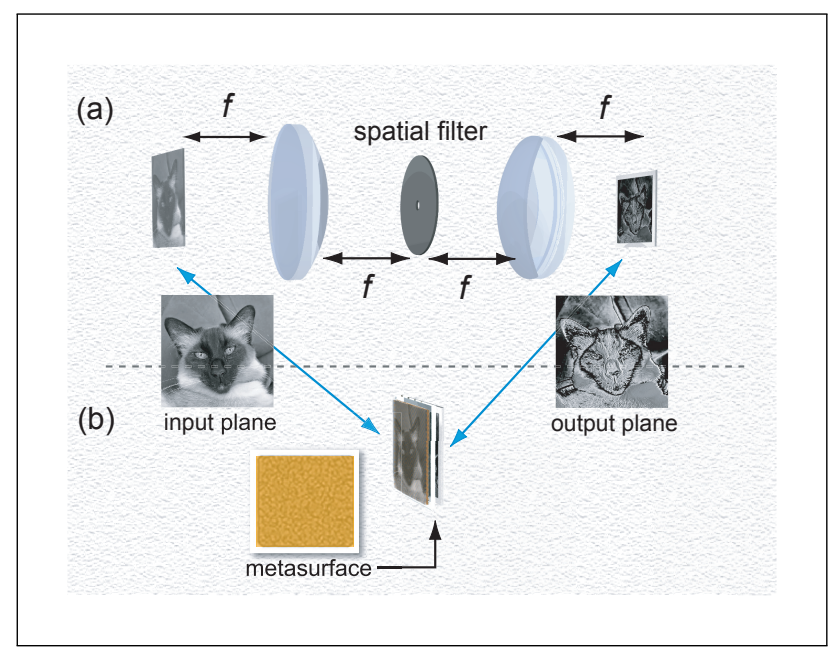

Fig. 1. The concept of a metasurface as an optical image processing element. (a) a standard 4- $f$ imaging system used for optical information processing; and (b) the concept under consideration here where the spatial filtering properties of the metasurface alters the image contrast. 
configuration has been demonstrated for wavefront retrieval [22] and image edge enhancement [5] using the dependence of surface plasmon polariton (SPP) excitation on angle of incidence. This approach has the advantage of removing any need for a Fourier transforming lens, but still requires the use of a prism to excite SPPs. Metasurfaces exhibit various resonances and have also been proposed as methods for information processing [3, 5]. Demonstrations, however, have focused on devices that can be used as spatial filters in a conventional optical information processing arrangement, albeit one where there is considerable flexibility in the design of the spatial filter. Metasurfaces consisting of nanoscale gold 'dolmen' structures have been shown to generate an angle-sensitive cross-polarized component in the transmitted field, enabling phase gradients in an incident field to be measured experimentally $[7,23,24]$ and light fields to be modulated optically [25]. Most recently, the use of resonant, subwavelength gratings as spatial differentiators [26, 27] and their application to edge detection [27] have been demonstrated.

Here we investigate the role of mode resonances in metasurfaces for manipulating the angular spectrum content of an incident field. It is well-known that certain modes of metasurfaces cannot be excited by a normally incident plane wave. These 'dark' modes, however, respond to illumination with non-zero transverse spatial frequencies and it is this approach to optical information processing that is investigated here (Fig. 1).

Below we develop a general formalism based on optical transfer functions to describe the properties of an angular or spatial frequency filter and its effect on an arbitrary incident light field. We demonstrate the use of this formalism utilizing two resonant plasmonic systems, although the method is general and is applicable to all-dielectric structures or combinations of dielectrics and plasmonics. Finally, we provide examples showing its application to high-pass filtering of amplitude and phase images.

\section{THEORETICAL BACKGROUND}

We first consider the interaction of an object of interest with an incident plane wave and decompose the resulting angular spectrum of the diffracted field into orthogonal polarization components. This field then interacts with a metasurface modifying this angular spectrum and, hence, the intensity of the resulting reflected or transmitted field.

Specifically, we consider a monochromatic plane wave with wavelength $\lambda$ and wavevector $\mathbf{k}_{0}=\left(k_{0 x}, k_{0 y}, k_{0 z}\right)$, where $k_{0 z}=$ $\sqrt{k_{0}^{2}-k_{0 x}^{2}-k_{0 y}^{2}}$ and $k_{0}=2 \pi / \lambda$, incident on an optically thin object of interest with transmission function $t(x, y)$. In the Kirchoff approximation, a scalar field transmitted through the object is given by the product of the incident field and $t(x, y)$. For plane wave illumination, therefore, diffraction by the object produces additional plane wave components with wavevector $\mathbf{k}=\left(k_{x}, k_{y}, k_{z}\right)$ where $k_{z}=\sqrt{k_{0}^{2}-k_{x}^{2}-k_{y}^{2}}$, and a field with an angular spectrum given by $T\left(k_{x}-k_{0 x}, k_{y}-k_{0 y}\right)$ where $T\left(k_{x}, k_{y}\right)$ is the Fourier transform of $t(x, y)$. This field is then transmitted through or reflected from a metasurface that consists of an infinite two-dimensional periodic array of subwavelength elements. We assume the transmission through (or reflection from) the metasurfaces of only a single propagating diffracted order. However, the amplitude of this plane wave (in general) depends on the angle of incidence. As a result, certain angular frequencies $\left(k_{x}, k_{y}\right)$ are preferentially transmitted or reflected modifying the angular spectrum of the scattered field and, hence, the in- tensity image. However, given the polarization sensitivity of metasurface modes, we need to also consider polarization in this analysis. A plane wave can be decomposed into TE (s) and TM $(p)$ polarized components (or any relevant polarization basis) so that its amplitude can be written:

$$
\tilde{\mathbf{E}}_{0}=\left(\begin{array}{c}
E_{0 s} \\
E_{0 p}
\end{array}\right),
$$

and the angular spectrum of the field diffracted by the object of interest (assumed non-birefringent) can then be written in the form

$$
\tilde{\mathbf{E}}_{t}\left(k_{x}, k_{y}\right)=T\left(k_{x}-k_{0 x}, k_{y}-k_{0 y}\right) \overline{\mathbf{P}}\left(k_{x}, k_{y}\right) \tilde{\mathbf{E}}_{0},
$$

where $\tilde{\mathbf{E}}_{t}\left(k_{x}, k_{y}\right)$ is similarly expressed in terms of $p$ and $s$ components defined relative to the plane of propagation defined by $\left(k_{x}, k_{y}\right)$. The matrix $\overline{\mathbf{P}}\left(k_{x}, k_{y}\right)$ describes changes in polarization of each scattered plane wave and is of the form, [28]

$$
\overline{\mathbf{P}}(\mathbf{k})=\left(\begin{array}{cc}
\Psi_{s S} & \Psi_{s p} \\
\Psi_{p s} & \Psi_{p p}
\end{array}\right) .
$$

where the elements of $\overline{\mathbf{P}}$ are given by

$$
\begin{aligned}
& \Psi_{s s}=\Psi_{p p}=\sqrt{\frac{k_{0 z}}{k_{z}}} \frac{\left(\mathbf{k}_{0} \cdot \mathbf{k}-k_{0 z} k_{z}\right)}{\sqrt{k_{0 x}^{2}+k_{0 y}^{2}} \sqrt{k_{x}^{2}+k_{y}^{2}}}, \\
& \Psi_{s p}=-\Psi_{p s}=\sqrt{\frac{k_{0 z}}{k_{z}}} \frac{\hat{z} \cdot\left(\mathbf{k}_{0} \times \mathbf{k}\right)}{\sqrt{k_{0 x}^{2}+k_{0 y}^{2}} \sqrt{k_{x}^{2}+k_{y}^{2}}} .
\end{aligned}
$$

These expressions arise because the polarization vector components change on scattering and the electromagnetic plane wave must remain transverse (Fig. 2 b). Note that at normal incidence, $k_{0 x}=k_{0 y}=0, k_{0 z}=k_{0}$, and the polarization matrix takes the form

$$
\overline{\mathbf{P}}(\mathbf{k})=\frac{1}{\sqrt{k_{x}^{2}+k_{y}^{2}}} \sqrt{\frac{k_{0}}{k_{z}}}\left(\begin{array}{cc}
k_{x} & -k_{y} \\
k_{y} & k_{x}
\end{array}\right),
$$

and we recover the results of Mansuripur [28] if we take $x$ polarization at normal incidence to correspond to $p$-polarized illumination and $y$-polarization to $s$.

Now that the behavior of the field refracted or scattered by an object has been decomposed into orthogonal polarization components, we can consider the action of a spatially dispersive but homogeneous thin film on the transmitted (or reflected) field. The influence of the metasurface is characterized by its optical transfer function (OTF), a tensor $\overline{\mathbf{H}}\left(k_{x}, k_{y}\right)$ that maps the incident field to the transmitted (or reflected) field including the polarization properties of the light beam:

$$
\overline{\mathbf{H}}\left(k_{x}, k_{y}\right)=\left(\begin{array}{ll}
H_{s S}\left(k_{x}, k_{y}\right) & H_{s p}\left(k_{x}, k_{y}\right) \\
H_{p s}\left(k_{x}, k_{y}\right) & H_{p p}\left(k_{x}, k_{y}\right)
\end{array}\right),
$$

where the subscripts $p$ and $s$ refer to orthogonal states of incident and scattered polarization. The off-diagonal elements of $\overline{\mathbf{H}}$ correspond to cross-polarization terms. The metasurfaces will modify or filter the spatial frequency components of the incident field and the transmitted field will take the form

$$
\tilde{\mathbf{E}}_{f}\left(k_{x}, k_{y}\right)=\overline{\mathbf{H}}\left(k_{x}, k_{y}\right) \tilde{\mathbf{E}}_{t}\left(k_{x}, k_{y}\right) .
$$




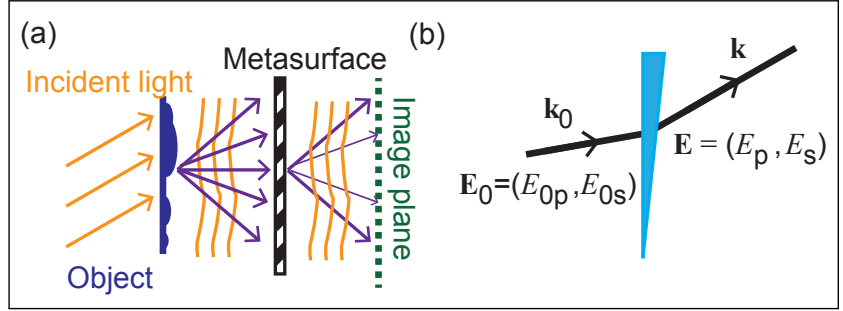

Fig. 2. (a) Concept showing image processing using angular sensitive surfaces. A plane wave is incident on an object containing information of interest. The scattered field can be expressed in terms of its angular spectrum which is modified by a metasurface exhibiting spatial dispersion. Note that the distances between the object, metasurface and image plane have been exaggerated for clarity. (b) Schematic showing change in direction of an angular spectrum component and polarization on refraction.

Hence the angular spectrum of the final filtered field $\tilde{\mathbf{E}}_{f}$, can be simply expressed as the product of terms relating to the incident illumination $\tilde{\mathbf{E}}_{0}$, the Fourier spectrum of the object or image and the properties of the metasurface or other film. This result then permits determination of the optical field as it propagates away from the metasurfaces or through a general optical system. If the transmission through the metasurface exhibits spatial dispersion, i.e. a dependence on the angle of incidence, this leads to Fourier filtering of the incident field providing a mechanism for direct 'spatial frequency filtering' without the requirement for additional lenses to transform the field to the Fourier plane. Note that Eq. (7) involves the vector field amplitudes rather than their intensity and, hence, it would be expected that the transmitted intensity (in the spatial domain, which is proportional to $\left|E_{f}\left(k_{x}, k_{y}\right)\right|^{2}$ ) could have a dependence on the phase of the scattered or transmitted field $t(x, y)$. Indeed, such a dependence leads to phase-contrast in the final image. Note that above we have precluded any additional phase contributions as a result of propagation from the object to the spatially dispersive film, although this could be included if important.

Eq. (2)-Eq. (7) encapsulate the key results from this paper and below we investigate the role that the excitation of different modes of metasurfaces could have on the transmitted field. It is apparent that the calculation of the components of the transfer tensor $\overline{\mathbf{H}}$ of the metasurface underpins the design of the structure. To investigate specific metasurface geometries, one can use full-field solvers such as the Finite Element Method, or the Finite-Difference Time-Domain Method to determine its angle and polarization response. There are, however, other methods requiring approximations or limitations in applicability that have a greater capacity to provide insight into the behavior of the device under consideration. Here we utilize two of these.

\section{METASURFACE TRANSFER FUNCTIONS}

The unit cell of a metasurface can be treated as a single resonant element that supports a number of radiant and subradiant modes. This is true whether the unit cell consists of a single, continuous entity or an ensemble of elements. In the latter case, it is also possible to determine the properties of the metasurface by looking at the modes of individual particles and their intra-cell coupling. Here, we use both approaches to study the response to spatial frequency of different types of metasurfaces.
Note that the approach described below is quite general, but its validity will depend on the details of the specific modeling method adopted and its underlying assumptions. In subsequent sections we use the framework described in Section 3A in conjunction with the electrostatic approximation and the modal method applied to a perfectly conducting frequency-selective surface.

\section{A. General Properties}

The response of the metasurface to an incident wave and the subsequent scattering can be described in general terms using the natural resonances or modes of the metasurface constituents. The fields $\chi$ in the metasurface or other film are represented by a linear combination of modes

$$
\chi=\sum_{n} a_{n} \psi_{n}
$$

that involves the eigenfunctions $\psi_{n}$ describing mode $n$ within the unit cell. These eigenfunctions may represent the standing waves within the unit cell of the metasurface, waveguide modes of apertures in metallic films, resonances within the periodic region of a diffraction grating or even localized surface plasmon modes. The expansion coefficient $a_{n}$ represents the response of the modes when the unit cell is placed in an array and therefore includes the interactions between cells.

In a similar fashion, the fields above the metasurface and in the supporting substrate can be written as a Floquet expansion of the form

$$
\Phi=\sum_{n \alpha} E_{n}^{\alpha} \phi_{n}^{\alpha}
$$

where the spatial distributions of the Floquet modes $\phi_{n}^{\alpha}$ are characterized by the index $n$ defining the direction of propagation and the polarization by $\alpha$. The Floquet modes represent plane waves constrained by the periodicity of the metasurface.

The expansion coefficient $a_{n}$ of Eq. (8) is found using the usual theory of orthogonal functions. However, it is often more convenient to first find the amplitude $b_{n}$ of each eigenmode of an isolated unit cell or resonant component in a unit cell and then derive $a_{n}$ from the inter- or intra-cell coupling. The overlap between the eigenfunction $\psi_{n}$ and the zero-th order Floquet mode of the incident field $\phi_{0}^{\alpha}$ is related to the input impedance, where the input coupling factor is $\gamma_{I}^{\alpha}$. The coupling constant is derived from the boundary conditions that relate the Floquet modes (incident and scattered) to the modes of the structure. These Floquet modes can then be expanded in terms of the internal modes or eigenfunctions of the metasurface, with expansion coefficients given by

$$
b_{n}=\sum_{\alpha} E_{0}^{\alpha} C_{n}^{0 \alpha}
$$

with

$$
C_{n}^{0 \alpha}=\int \gamma_{I}^{0 \alpha} \psi_{n}^{*} \phi_{0}^{\alpha} d V
$$

The integral is over the unit cell of the metasurface and accommodates the thickness of the structure.

Each excited mode within a unit cell of the metasurface may couple to modes within a unit cell or between cells. For example, a localized surface plasmon (LSP) mode on a nanoparticle in the unit cell may excite an LSP mode on an adjacent particle. This coupling is described by a matrix coefficient $G_{n m}$ coupling mode $m$ with mode $n$. When we solve for the amplitudes $a_{n}$ in the coupled system, in terms of the uncoupled mode amplitudes $b_{n}$, we find they depend on the inverse of the coupling matrix 
(see for example [29]). Then the resultant excitation leading to scattering of the incident light is a linear combination of coupled modes with amplitudes

$$
a_{n}=\sum_{m}\left[G^{-1}\right]_{m n} b_{m}
$$

which depends on the mode coupling coefficient $G_{m n}$. Examples of how this arises in practice will be given in the following section. The zeroth-order scattered field with polarization $\beta$ is then the contribution of all those modes that couple out of the metasurface back into free space

$$
\begin{aligned}
E_{0}^{\beta} & =\gamma_{0}^{\beta} \sum_{n} a_{n}\left[C_{n}^{0 \beta}\right]^{*} \\
& =\gamma_{0}^{\beta} \sum_{m n}\left[G^{-1}\right]_{m n} b_{m}\left[C_{n}^{0 \beta}\right]^{*} \\
& =\gamma_{0}^{\beta} \sum_{m n \alpha}\left[G^{-1}\right]_{m n} C_{m}^{0 \alpha}\left[C_{n}^{0 \beta}\right]^{*} E_{0}^{\alpha},
\end{aligned}
$$

with the out-coupling coefficient $\gamma_{0}^{\beta}$, related to the impedance, derived again from the boundary conditions. Comparing this expression with Eq. (7) it is apparent that the metasurface optical transfer function can then be written as

$$
H_{\beta \alpha}=\gamma_{0}^{\beta} \sum_{m n}\left[C_{n}^{0 \beta}\right]^{*}\left[G^{-1}\right]_{m n} C_{m}^{0 \alpha},
$$

which depends on how the fields external to the metasurface couple to the resonant elements $\left(C_{m}^{0 \alpha}\right)$, how these elements couple to each other $\left(\left[G^{-1}\right]_{m n}\right)$ and how the subsequent modes couple out again $\left(\left[C_{n}^{0 \beta}\right]^{*}\right)$ into free space or subsequent elements in an optical system.

The details contained in this general formalism depend on the theoretical formulation used. Two examples of this general form are discussed below - the Electrostatic Eigenmode Method (Section B) which has been shown to be a powerful tool in investigating metasurfaces when the unit cell consists of ensembles of nanoparticles, and the Modal Method (Section C) that originated in studies of frequency-selective surfaces. [30,31] Generally, the overlap integral contains information about the spatial frequencies of the incident wave and the spatial filtering properties of the metasurface.

\section{B. Electrostatic eigenmode method}

An analysis of the response of sub-wavelength arrays of metal nanoparticles supporting localized surface plasmon (LSP) resonances can be performed using the Electrostatic Eigenmode Method (EEM) [29]. This approximate algebraic method is based on a representation of the LSP surface charge in terms of the fundamental eigenmodes of each metal nanoparticle. For simplicity, we consider only a single LSP eigenmode $\sigma_{n}(\mathbf{r})$ on each nanoparticle $n$ so that the total LSP surface charge in a unit cell induced by the incident field is given by

$$
\sigma(\mathbf{r})=\sum_{n} a_{n} \sigma_{n}(\mathbf{r})
$$

This is equivalent to Eq. (8) of the previous section. In the absence of coupling between particles or when there is an isolated nanoparticle in the unit cell, we have $a_{n}=b_{n}$. A plane wave incident on metal nanoparticle $n$ located at $\mathbf{r}_{n}$ is already in the Floquet form $E_{0}^{\alpha} \exp \left(i \mathbf{k} \cdot \mathbf{r}_{n}\right)$ (compare with Eq. (9)). The surface charge is found from the boundary conditions on the components of the electric displacement and the electric field across the metal surface, as discussed in [29]. To extract out the coefficient $a_{n}=b_{n}$ in the absence of coupling, we multiply the resulting expression for the surface charge by the adjoint eigenfunction $\psi_{n}^{*}=\tau_{n}$, which, for technical reasons in the EEM is the surface dipole distribution $\tau_{n}(\mathbf{r})$ [29]. Then the expansion coefficient is given by an integral over the nanoparticle surface at $\mathbf{r}_{n}+\mathbf{r}$

$$
C_{n}^{0 \alpha} E_{0}^{\alpha}=f_{n}(\omega) \oint \tau_{n}\left(\mathbf{r}-\mathbf{r}_{n}\right) \hat{n}^{\alpha}(\mathbf{r}) E_{0}^{\alpha} \exp \left(i \mathbf{k} \cdot\left(\mathbf{r}_{n}+\mathbf{r}\right)\right) d S,
$$

which is equivalent to Eq. (11). The factor $f_{n}(\omega)$ is the dipole moment per unit volume of the metal nanostructure. It is frequency dependent and determines the shape of the scattering spectrum and the phase of the LSP oscillation relative to the applied field. The factor $\hat{n}^{\alpha}(\mathbf{r})$ is the vector component in direction $\alpha$ of the nanoparticle surface normal at position $\mathbf{r}$. The product $f_{n} \hat{n}^{\alpha}(\mathbf{r})=\gamma_{I}^{0 \alpha}$ is the input coupling factor. If the nanoparticle is much smaller than the wavelength of light, the incident electric field is approximately constant across its surface and can be removed from the integral, leaving a term equivalent to the total dipole moment of the eigenmode of the nanoparticle $n$,

$$
p_{n}^{\alpha}=\oint \tau_{n}\left(\mathbf{r}-\mathbf{r}_{n}\right) \hat{n}^{\alpha}(\mathbf{r}) d S .
$$

This equation leads to a simplified expression $C_{n}^{0 \alpha}=$ $f_{n}(\omega) p_{n}^{\alpha} \exp \left(i \mathbf{k} \cdot \mathbf{r}_{n}\right)$.

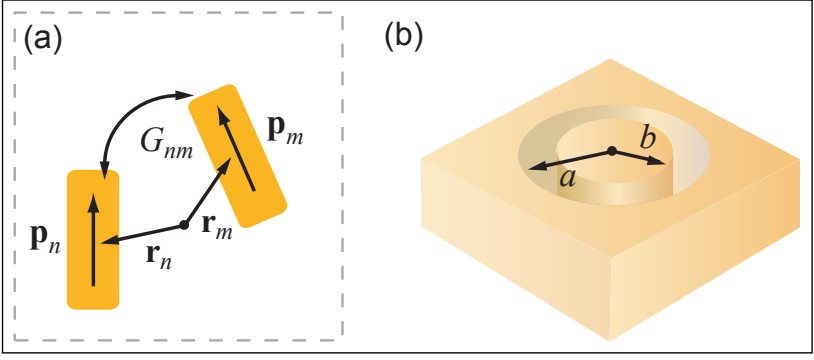

Fig. 3. (a) The geometry of a unit cell of plasmonic nanoparticles showing the distance vectors $\mathbf{r}_{n}$ to two of the nanoparticles, their dipole moments $\mathbf{p}_{n}$ and the coupling between them $G_{n m}$; (b) The geometry of the aperture in a thick perfectly conducting film.

When there is more than one nanoparticle in a unit cell (Fig. $3 \mathrm{a}$ ), or if there is coupling between cells, the resonant modes are altered so that $a_{n} \neq b_{n}$. The new modes can be expressed as a linear combination of the old modes which takes the form $a_{n}=\sum_{m}\left[G^{-1}\right]_{m n} b_{m}$ where $G_{n m}(\omega)$ is the frequencydependent coupling between the LSP on nanoparticle $m$ and that on nanoparticle $n$. The sum is over all particles that are involved in the coupling. Since the derivation is involved we do not present it here (see Ref. [29] for details) but it can be shown that the coupled amplitudes depend on the inverse of $G_{n m}(\omega)$. The resulting dipole moment of particle $n$ is then $a_{n} p_{n}^{\beta}$. The dipole radiation scattered into the far-field from the LSP dipole moments of the metal nanoparticles is given by the usual expression [32]

$$
E_{0}^{\beta}=\frac{k^{2}}{4 \pi \epsilon_{0}} \sum_{n} a_{n}\left(p_{n}^{\beta}-\hat{n}^{\beta}\left(\sum_{\delta} p_{n}^{\delta} \hat{n}^{\delta}\right)\right) \frac{e^{i k R_{n}}}{R_{n}},
$$

where $R_{n}=\left|\mathbf{r}-\mathbf{r}_{n}\right|$ is the distance from nanoparticle $n$ to the point of observation and $\hat{n}$ is the unit vector in the direction of scattering. The distance can be approximated by $R_{n} \approx r-\hat{n} \cdot \mathbf{r}_{n}$ 
since $r \gg r_{n}$. Combining these terms together leads to an expression for the electric field scattered from the coupled metal nanoparticles

$$
E_{0}^{\beta} \approx \frac{k^{2} \exp (i k r)}{4 \pi \epsilon_{0} r} \sum_{m n \alpha} q_{n}^{\beta}\left[G^{-1}\right]_{m n} f_{m} e^{i \mathbf{k} \cdot\left(\mathbf{r}_{m}-\mathbf{r}_{n}\right)} p_{m}^{\alpha} E_{0}^{\alpha},
$$

where for convenience we have written $q_{n}^{\beta}=p_{n}^{\beta}-\hat{n}^{\beta}\left(\sum_{\delta} p_{n}^{\delta} \hat{n}^{\delta}\right)$.

In terms of the general treatment in the previous section (sec. 2) we identify $\gamma_{0}^{\beta}=k^{2} \exp (i k r) / 4 \pi \epsilon_{0} r,\left[C_{n}^{0 \beta}\right]^{*}=q_{n}^{\beta} \exp (-i \mathbf{k}$. $\left.\mathbf{r}_{n}\right)$ and $C_{m}^{0 \alpha}=f_{m} \exp \left(i \mathbf{k} \cdot \mathbf{r}_{m}\right) p_{m}^{\alpha}$ so that the metasurface optical transfer function is

$$
H_{\beta \alpha}(\mathbf{k})=\gamma_{0}^{\beta} \sum_{n m} q_{n}^{\beta}\left[G^{-1}\right]_{m n} f_{m} e^{i \mathbf{k} \cdot\left(\mathbf{r}_{m}-\mathbf{r}_{n}\right)} p_{m}^{\alpha} .
$$

A metasurface created by an array of these coupled metal structures (with subwavelength periodicity) will behave like a thin film with a spatial dispersion described by $H_{\beta \alpha}(\mathbf{k})$, where the spatial-frequency dependence arises from the linear combination of phase terms $e^{i \mathbf{k} \cdot\left(\mathbf{r}_{m}-\mathbf{r}_{n}\right)}$. Such a metasurface will modify the Fourier components of any incident beam leading to spatial frequency filtering, in amplitude, phase or both. If the configuration of metal structures has a dark mode (i.e. $\mathbf{p}_{m}=0$ ), then this will not be excited at $\mathbf{k}=0$ since the incident light is predominantly dipolar in nature leading to no LSP excitation and no subsequent effect on the undeviated beam. However, light incident at off-normal incidence has an effective quadrupole component associated with the phase shift due to the tilt of the beam. Such a beam can excite dark modes that have a non-zero quadrupole moment, resulting in a change in the optical transfer function and therefore spatial filtering. That is, the phase shift across the structure of the tilted incident beam is sufficient to excite the mode.

\section{Modal methods}

The other technique that we will use for illustrating the metasurface OTF involves a structure that consists of an array (period d) of apertures in a thick, perfectly electrically conducting (PEC) metal film or an array of infinitesimally thin PEC scatterers (Fig. 3 b). As discussed in detail elsewhere [30, 31, 33-35], we can write the fields within the apertures, or the currents on the scatterers, as a sum over the corresponding waveguide (in the case of holes) or current (in the case particles) modes and the fields above the structure and in the substrate as a discrete sum over plane waves satisfying the Floquet condition. The relevant boundary conditions can then be used to derive a set of linear equations for the mode amplitude $a_{n}$. These can be found by solving the set of linear equations of the form given by Eq. (10) and Eq. (12). In this case $G$ is the matrix that describes coupling of the Floquet to waveguide or scatterer modes and $C_{m}^{\alpha}$ is the overlap integral between the incident plane wave with polarization $\alpha$ and cavity or scatterer mode $m$, i.e. it describes the coupling constant between the incident field and the modes. For apertures in thick films, two sets of coupled equations are required to determine the amplitudes of upward and downward traveling modes in the apertures [30, 31, 34, 35]. The amplitude of the scattered, zero-th order, plane wave with polarization $\beta$ is then given by

$$
E_{0}^{\beta}=Z_{\beta} \sum_{n} a_{n}\left(C_{n}^{0 \beta}\right)^{*}=Z_{\beta} \sum_{m n \alpha}\left[G^{-1}\right]_{m n} C_{n}^{0 \alpha}\left(C_{n}^{0 \beta}\right)^{*} E_{0}^{\alpha},
$$

with

$$
Z_{p}=Z_{0} \frac{k_{0 z}}{k_{0}}
$$

and

$$
Z_{s}=Z_{0} \frac{k_{0}}{k_{0 z}},
$$

where $Z_{0}$ is the impedance of free-space and $k_{0 z}$ is the $z$ component of the incident wavevector. Note that Eq. (22) is identical in form to Eq. (13). Hence, the elements of the transfer function of Eq. (6) are given by:

$$
H_{\beta \alpha}=Z_{\beta} \sum_{m n}\left[G^{-1}\right]_{m n} C_{n}^{0 \alpha}\left(C_{n}^{0 \beta}\right)^{*} .
$$

In the case where only one mode is resonant, and particularly for transmission through inductive grids, reasonable convergence can be obtained by including only this mode in a monomodal calculation. [36] In this case $H_{\beta \alpha} \propto Z_{\beta} C_{\alpha} C_{\beta}^{*}$. This means the strength of the coupling coefficients $C_{\alpha}$ provides information about the elements of the transfer function. As in Section 3B, it is also instructive to consider metasurfaces where the unit cell consists of several dipole scatterers. In these cases, the sums appearing in Eq. (21) and Eq. (24) extend over each scatterer as well as their modes.

\section{CASE STUDIES}

\section{A. Plasmonic Wheatstone bridge (dolmen)}

Three nano rods can be arranged in a "dolmen" configuration and combined with crossed polarizers to create a metasurface high-pass filter. [7, 23] This configuration consists of two parallel rods and a third rod at right angles coupling the two (Fig. 4 (a)). Since the two parallel rods couple to opposite ends of the third rod and we assume the coupling magnitude is the same, we can write $G_{13}=-G_{23}=G$. This structure mimics the Wheatstone Bridge in electrical systems and responds to the difference between the light waves incident on the two parallel rods. Light incident on each of the two rods polarized parallel to their long axes induces surface plasmons with opposite phases in the third rod, which subsequently cancel out. An imbalance between the amplitudes or phases on the two parallel rods leads to incomplete cancellation and a residual LSP generated in the third rod, which radiates light polarized perpendicular to the incident light.

Using crossed polarizers enables the OTF for this configuration to be found by writing the dipole moments of the rods as $\mathbf{p}_{1,2}=p \hat{y}$ and $\mathbf{p}_{3}=p \hat{x}$ with the two parallel rods located at $\mathbf{r}= \pm d \hat{x} / 2$ and the third rod at $\mathbf{r}_{3}=h \hat{y}$. If we let $\alpha, \beta \in\{\hat{x}, \hat{y}\}$, then the crossed polarizers result in $H_{y x}=H_{x x}=H_{y y}=0$ leaving the only term in Eq. (20)

$$
H_{x y}(\mathbf{k})=\gamma_{0}^{x}\left(\frac{2 i p^{2} f^{2}(\omega) G e^{-i k_{y} h}}{1-2 f(\omega)^{2} G^{2}}\right) \sin \left(k_{x} d / 2\right)\left(1-\left(k_{x} / k\right)^{2}\right),
$$

where all rods have the same magnitude of the dipole moment $p$ and the same resonance factor $f(\omega)$. The various terms in Eq. (25) relate to those in Eq. (20) after summing over the nanoparticles as follows: $q_{n}^{\beta} \rightarrow p\left(1-\left(k_{x} / k\right)^{2}\right),\left[G^{-1}\right]_{m n} \rightarrow$ $(2 i f(\omega) G) /\left(1-2 f(\omega)^{2} G^{2}\right), e^{i \mathbf{k} \cdot\left(\mathbf{r}_{m}-\mathbf{r}_{n}\right)} \rightarrow \sin \left(k_{x} d / 2\right) e^{-i k_{y} h}$ and $p_{m}^{\alpha} \rightarrow p$. Details on the derivation of the coupling can be found elsewhere [8, 37]

The expression Eq. (25) shows that the magnitude of the OTF depends only on $k_{x}$ since the $k_{y}$ dependence only contributes an irrelevant phase shift. In practice, the first polarizer would be placed on top of the metasurface so that unpolarized light incident at any angle always has the $E_{x}$ component removed. 


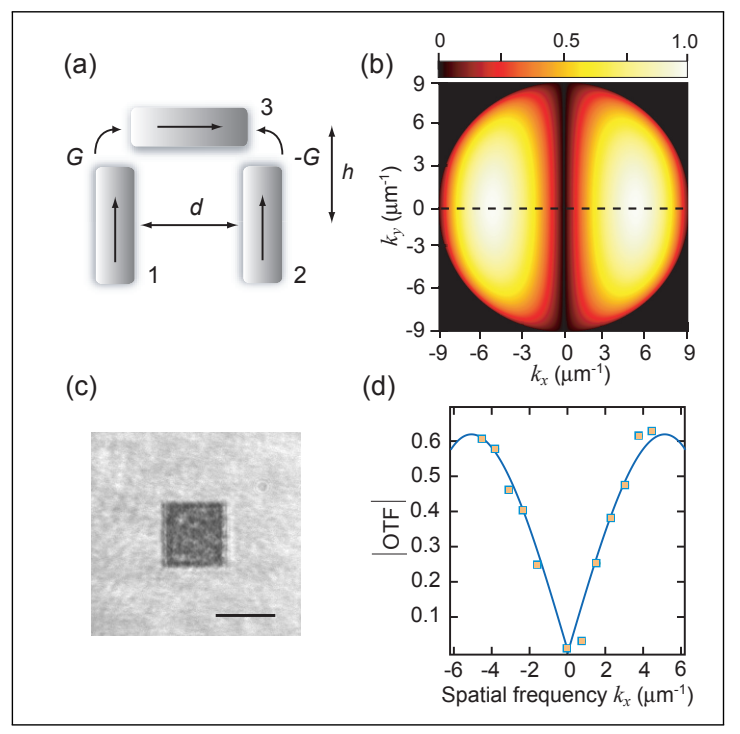

Fig. 4. a) The Wheatstone Bridge configuration showing the relative directions of the induced dipole moments when the light incident on rods 1 and 2 is unbalanced. The coupling coefficient is such that $G_{13}=-G_{23}=G$; b) The magnitude of the optical transfer function $\left|H_{x y}\right|$ calculated using Eq. (25) and Eq. (26); c) A microscope image of a metasurface made from gold WB structures with a period of $400 \mathrm{~nm}$. The scale bar is $100 \mu \mathrm{m} ; \mathrm{d}$ ) Calculated (solid line) and measured (points) of the magnitude of the optical transfer function of the metasurface in (c) taken along the dashed line in (b). The experimental data was adapted from [7].

However, the field that passes through the polarizer will vary between $s$ polarization and $p$ polarization depending on the angle of incidence so that the incident amplitude will vary according to

$$
\mathbf{E}_{t} \cdot \hat{y}=E_{t} \frac{\sqrt{k^{2}-k_{x}^{2}-k_{y}^{2}} \sqrt{k_{x}^{2}+k_{y}^{2}}}{\sqrt{\left(k^{2}-k_{x}^{2}-k_{y}^{2}\right) k_{x}^{2}+k^{2} k_{y}^{2}}}
$$

where $k^{2}=k_{x}^{2}+k_{y}^{2}+k_{z}^{2}$. Including this factor in the transfer function of Eq. (25) yields the result in Fig. 4 (b) where $d=$ $140 \mathrm{~nm}, \lambda=700 \mathrm{~nm}$ (and therefore $k=8.98 \mu \mathrm{m}^{-1}$ ) and the magnitude was normalized.

In this instance, experimental verification of the derived OTF can be obtained from previously published data [7] on the phase sensitivity of a metasurface created from arrays of Wheatstone bridge structures made from gold nanorods on glass. A $100 \mu \mathrm{m}$ square metasurface (Fig. $4 \mathrm{c}$ ) is illuminated at different incident angles with collimated white light passed through a polarizer. The light emerging from the metasurface is filtered by a cross-polarizer and the intensity $I_{f}$ measured (see [7] for details). The magnitude of the OTF is proportional to $\sqrt{I_{f}}$ as shown in Fig. 4 (d) where a background offset has been removed. A one-parameter fit of the theoretical OTF is performed to set the vertical scale which shows very good agreement with experimental values. As we show in the Appendix, a metasurface with subwavelength periodicity behaves, to first order in scattering, like a uniform thin film.

\section{B. Annular apertures in a PEC film}

The particular geometry under consideration here (Fig. 5 (a)) has a unit cell containing a coaxial aperture with inner radius $b$ and outer radius $a$. Using the modal method described in 3C above, this structure has been shown to exhibit strong bright, dipole modes as well as resonances associated with the excitation of the Transverse Electromagnetic Mode (TEM) which is subradiant. [38] The resonance frequencies of the various modes can be tuned by modifying the geometry of the aperture and the thickness of the metal film. In terms of the transport of power through the structure, the most important modes at normal incidence are the zero-th and higher order Fabry-Perot (FP) TE 11 modes. At off-normal incidence, for $p$-polarization, the first and higher order F-P modes of the TEM mode can be excited. This is a dark (subradiant) mode and cannot be excited with a normally incident plane wave.

One of us [39] investigated the role that the $\mathrm{TE}_{11}$ and TEM modes play in the transmission of finite beams through the structure and the research presented here generalizes these results to arbitrary incident fields, specifically those arising from diffraction by an object. In this case the coupling to the modes of interest, which informs the contribution to transmission through the structure by each mode, depends on the coupling of the incident field to the mode of interest as encapsulated in the coefficients $C_{n}^{0 \alpha}$. The relevant coupling coefficients are found by calculating the projection of coaxial waveguide modes onto Floquet modes and explicit expressions are given in reference [31]. Here we investigate their dependence on the spatial frequencies of the incident field when the width of the rings $(a-b)$ is small $(a-b) \ll b / 2$ and $b \ll \lambda$. In this limit, the coefficient characterizing coupling between the TEM mode and a $p$-polarized plane wave has a magnitude given by

$$
\left|C_{\mathrm{TEM}}\left(k_{x}, k_{y}\right)\right| \approx \frac{\pi b(a-b) \sqrt{k_{x}^{2}+k_{y}^{2}}}{d \sqrt{2 \pi \ln (a / b)}} .
$$

In the case of an incident s-polarized plane wave, the coupling coefficient is zero. From this expression it is apparent that at normal incidence, a plane wave cannot excite this mode. The TEM mode is a dark mode. The other mode that makes a significant contribution in transporting energy through the structure is the $\mathrm{TE}_{11}$ mode which has a net electric dipole moment and is a bright mode. In the same limit, the coupling coefficient for this mode has a magnitude given by:

$$
\left|C_{\mathrm{TE} 11}\left(k_{x}, k_{y}\right)\right| \approx \frac{\pi(a-b)}{d}\left|\mathcal{Z}_{1}\left(c \chi^{\prime}\right)\right|,
$$

where $\mathcal{Z}_{1}$ is a first order combination Bessel-Neumann function defined for coaxial waveguides $[31,40]$ and $\chi^{\prime}$ is the lowest value satisfying $\mathcal{Z}_{1}\left(a \chi^{\prime} / b\right)=0$. Note that there are two degenerate $\mathrm{TE}_{11}$ modes with orthogonal dipole moments which are, therefore, bright modes. It is apparent that the TEM mode is sensitive to the transverse component of the incident wavevector. The transmitted power will therefore be proportional to $k_{x}^{2}+k_{y}^{2}$, producing a high pass filter where for a fixed gap width $(a-b)$, the sensitivity increases with the size of the ring. Note that the sensitivity to the angle of incidence depends on the geometry of the apertures. In the limit considered, the coupling to the $\mathrm{TE}_{11}$ mode described by Eq. (6), on the other hand, has no explicit dependence on the angle of incidence and would produce no significant spatial filtering. These observations are consistent with the findings of reference [39]. 


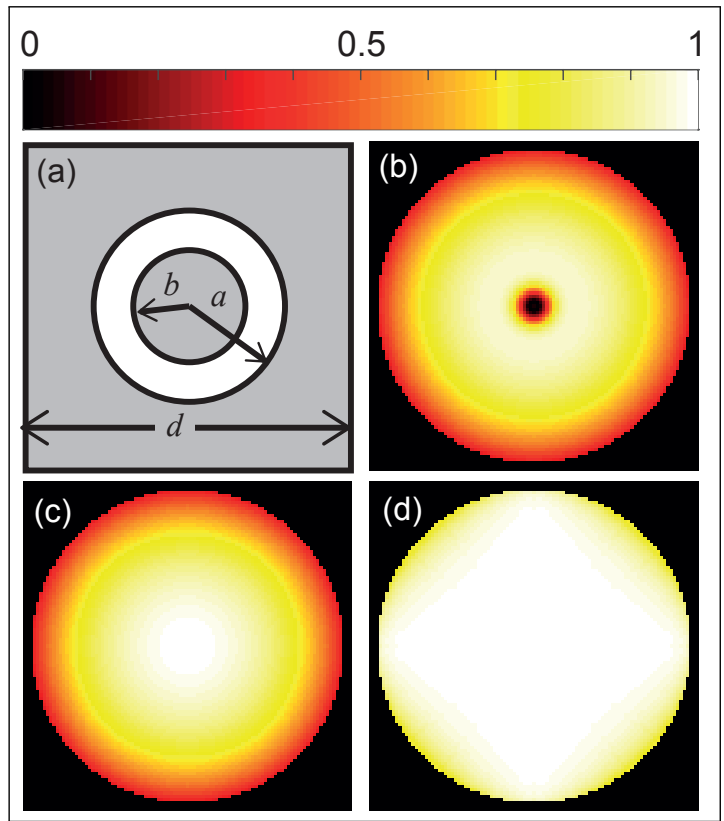

Fig. 5. Schematic (a) of the unit cell of an array of apertures in a perfectly conducting film with period $d$. The inner and outer ring radii are $0.40 d$ and $0.45 d$, and the thickness of the film is set to $1.5 d$. Figure (b) shows $\left|H_{p p}\right|$ at a wavelength of $3.167 d$ corresponding to a resonance of the TEM mode. Figures (c) and (d) show $\left|H_{p p}\right|$ and $\left|H_{s s}\right|$ at a wavelength of $2.650 d$ corresponding to a resonance of the $\mathrm{TE}_{11}$ coaxial waveguide mode. Normalized spatial frequencies plotted from -1 to 1 in (b), (c) and (d).

We revisit the specific geometry of [39] with $a=0.45 d$, $b=0.40 d$ and thickness $h=1.5 d$ where $d$ is the period of the array. This geometry was selected to provide spectral separation of the lowest order Fabry-Perot $\mathrm{TE}_{11}$ and TEM resonances. Fig. $5(b-d)$ shows the significant, propagating elements of the optical transfer function at different wavelengths. Fig. 5(b) shows $\left|H_{p p}\right|$ plotted at a wavelength of $3.167 d$, corresponding to the first order Fabry-Perot TEM coaxial cavity resonance. Fig. 5(c) and (d) show $\left|H_{p p}\right|$ and $\left|H_{s s}\right|$ at a wavelength of $2.65 d$ corresponding to a resonance of the $\mathrm{TE}_{11}$ mode. In both cases, the other components of the transfer function tensor are much weaker and are not shown. In the calculations of Fig. 5, $6 \mathrm{TE}((0,1),(1,1-3)$, $(2,1)$ and $(3,1))$ and 6 TM (as per TE) modes were used in the calculation and plane waves with normalized $z$-component of the wavevector up to 10.0 are included.

In Fig. 5(b) at the TEM mode resonance, a strong dependence on spatial frequency consistent with Eq. (28) is apparent. On the other hand at a wavelength where the bright mode dominates, Fig. 5(c) and (d) show only a weak dependence on spatial frequency. As discussed above, this mode is relatively robust to angle of incidence and there is little sensitivity to spatial frequency.

\section{IMAGE PROCESSING EXAMPLES}

As an illustration, the formalism of Eq. (1)-Eq. (5) is used to calculate the intensity of the field transmitted through either a Wheatstone bridge metasurface placed between crossed polarizers, or an annular aperture array illuminated with linearly polarized or unpolarized light. The characteristic modification of the resulting intensity produced by propagation through the metasurfaces of interest is demonstrated. Both amplitude and pure phase objects are considered.

In the first example, we calculate the effect of a metasurface of Wheatstone bridge structures on a pure phase image. An image was created with a range of phase shifts and phase gradients (Fig. 6 (a)), where the black regions correspond to $0^{\circ}$ and the white regions to $180^{\circ}$. The image was $100 \mu \mathrm{m}$ square. The OTF of the metasurface is shown in Fig. 4 (b). With a collimated beam passing at normal incidence through the phase object, the resulting image after the metasurface is shown in Fig. 6 (b). Since the OTF is zero at normal incidence, most of the image is dark. However, regions with large phase gradients correspond to waves propagating at non-normal angles, which also have higher spatial frequencies. These pass through the metasurface creating the edge-enhanced image. If the incident beam is offset from the normal by $10^{\circ}$, the result is to add a bias to the spatial frequencies so that a fraction of the un-phaseshifted beam passes through. This yields the image in Fig. 6 (c).

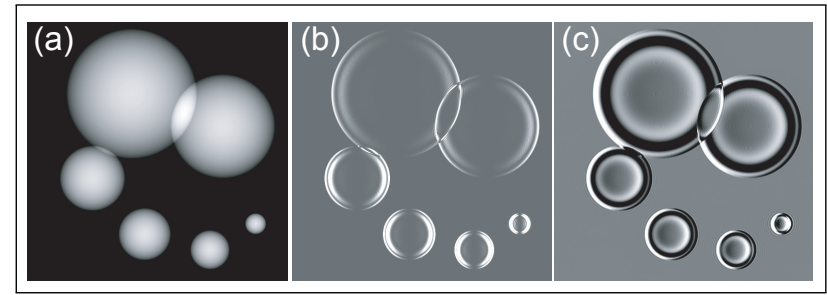

Fig. 6. Examples of imaging a phase object with a metasurface of Wheatstone bridge structures. a) Simulated phase object. The image is $100 \mu \mathrm{m}$ square with a maximum phase shift of $180^{\circ}$; b) the image after passing through the metasurface at normal incidence; $c$ ) the image with a $10^{\circ}$ tilt of the incident beam from normal in the $k_{x}$ direction.

The response of the coaxial aperture metasurface to the light transmitted through the phase-only object of Fig. 6(a) is considered in Fig. 7. If light, with a wavelength corresponding to a TEM mode resonance, is transmitted through the object and then passes through the aperture array, the intensity image of Fig. 7(a) is obtained. In the case of horizontally polarized light, the image of Fig. 7(b), results. As in the case of the dolmen structure, additional contrast can be obtained by introducing a phase bias along the direction of polarization. At an angle of incidence of $10^{\circ}$ so that $k_{0 x} \neq 0$, the resulting intensity image shown in Fig. 7(c) is obtained.

To demonstrate filtering of an amplitude image, a gray-scale image of a cat (Fig. 8 (a)) is passed through the coaxial aperture array at a wavelength corresponding to a resonance of the TEM mode and the transfer function of Fig. 5(b). Since only the $p$ component of incident light can excite the resonance, the filtering depends on the incident polarization. For unpolarized illumination, low spatial frequencies are simply suppressed and edges in the image enhanced (Fig. 8(b)). For horizontally polarized light (Fig. 8(c)) only high spatial frequencies along the horizontal are transmitted. This leads to relatively strong transmission of spatial frequencies carrying information about vertical lines in the image. This is apparent in Fig. 8(c), where the vertical edge of the wall and the edges of the cat's ears are enhanced. For vertical polarization, the strongest transmission occurs for higher spatial frequencies carrying information about horizontal lines. Note 


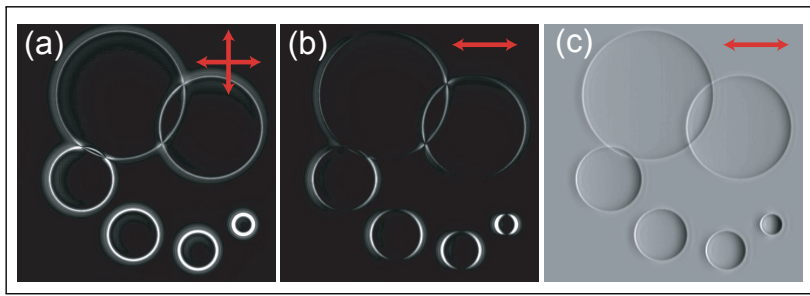

Fig. 7. Simulated images of a phase object (Fig.6 (a)) with a $\pi$ phase excursion produced after transmission via the TEM mode through the array of coaxial apertures with transfer function given in Fig. 4 (a). Illumination with normally incident, unpolarized light produces the intensity image shown in (a), whereas (b) and (c) show the resulting image obtained with horizontally polarized light at normal incidence (b) at an angle of $10^{\circ}$ to the normal (c). Image size is 500d and the wavelength is $3.167 d$.

that no evanescent spatial frequency components are included in these calculations. We assume that these are unresolvable or have decayed sufficiently before reaching the detector.
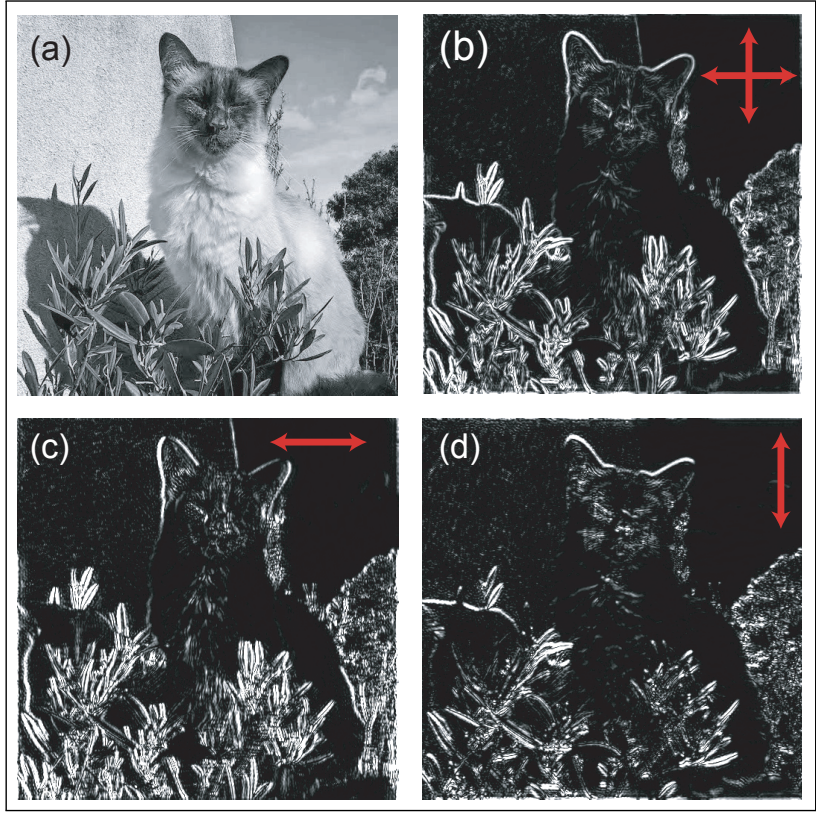

Fig. 8. Amplitude images transmitted via the TEM mode through an array of coaxial apertures as described in Fig. 4(a). If unpolarized light is used, the image shown in (b) results, whereas the images (c) and (d) correspond respectively to the use of horizontally and vertically polarized light. Image size is $500 d$ and the wavelength is $3.167 d$.

\section{CONCLUSION}

In conclusion, we have provided a framework to describe image transmission through uniform metasurfaces, including those exhibiting spatial dispersion. In the case where the dominant mode of interaction is dark or subradiant, the sensitivity to angle of incidence produces spatial frequency filtering. Analytic and numerical case studies involving metasurfaces where the unit cell consists of either an ensemble of metallic nanorods or an annular aperture in a perfectly electrically conducting film were presented and aspects of information and image processing presented. Their capacity to modify or enhance image contrast was illustrated, demonstrating their potential as elements in ultra-compact devices capable of on-chip, real-time, single-shot information processing.

\section{APPENDIX}

Metasurfaces by and large consist of periodic arrays of nanoscale structures that are constructed as identical unit cells repeated in two dimensions across the surface. Diffraction can arise when the periodicity $d$ of the array is of the order of the wavelength of light. In this supplement we derive the diffraction condition for a metasurface where $\lambda \gg d$ and show that a subwavelength structure with minimal inter-cell coupling behaves like a uniform thin film with properties determined by the structure of the unit cells. In essence, this framework excludes the interaction of diffracted evanescent fields with the metasurface.

In the weak-scattering approximation, the radiation scattered from each unit cell to another unit cell is weak or the scattering from all cells to a given cell combine out of phase and destructively interfere. The electric field incident on a unit cell at position $\mathbf{r}_{n}$ with an effective polarizability tensor $\bar{\alpha}$ induces a dipole moment $\mathbf{p}_{n}=\bar{\alpha} \cdot \mathbf{E}_{t} \exp \left(i \mathbf{k} \cdot \mathbf{r}_{n}\right)$. The polarizability tensor accounts for changes in the state of polarization on excitation, as often arises with plasmonic structures. The electric field of the radiation scattered from this dipole into the far-field has the dyadic form $\mathbf{E}_{n}=A \exp \left(i k R_{n}\right)(\mathbf{1}-\hat{n} \hat{n}) \cdot \mathbf{p}_{n}$ where the direction of scattering is $\hat{n}$ and $A$ is a constant. The distance $R_{n}=\left|\mathbf{r}-\mathbf{r}_{n}\right|$ from the dipole to the point of observation $\mathbf{r}$ can be approximated by $R_{n}=\sqrt{r^{2}+r_{n}^{2}-2 \mathbf{r} \cdot \mathbf{r}_{n}} \approx r-\hat{n} \cdot \mathbf{r}_{n}$ when $r \gg r_{n}$ where $\hat{n}$ is the unit vector in direction $\mathbf{r}$. The phase term is then $\exp \left(i k R_{n}\right) \approx \exp \left(i k r-i \mathbf{k}_{s} \cdot \mathbf{r}_{n}\right)$ where the wavevector in the direction of scattering is $\mathbf{k}_{s}=\hat{n} k$. For identical unit cells, the total scattered field $\mathbf{E}=A(\mathbf{1}-\hat{n} \hat{n}) \cdot \bar{\alpha} \cdot \mathbf{E}_{t} \sum_{n} \exp \left(i\left(\mathbf{k}-\mathbf{k}_{s}\right) \cdot \mathbf{r}_{n}\right)$. We write the scattering vector as $\mathbf{q}_{s}=\mathbf{k}_{s}-\mathbf{k}$ and assume the array has periodicity in two dimensions given by $\mathbf{r}_{n}=l d_{x} \hat{x}+m d_{y} \hat{y}$ where the sum over $n$ is divided over integers $l$ and $m$. This leads to

$$
\sum_{n} e^{i\left(\mathbf{k}-\mathbf{k}_{s}\right) \cdot \mathbf{r}_{n}}=\sum_{l=1}^{N_{x}} \sum_{m=1}^{N_{y}} e^{-i l \mathbf{q}_{s} \cdot \hat{x} d_{x}} e^{-i m \mathbf{q}_{s} \cdot \hat{y} d_{y}},
$$

where $N_{x}$ and $N_{y}$ are the number of unit cells in $x$ and $y$ with a total $N=N_{x} N_{y}$. The sums are standard geometric series that can be evaluated

$$
\begin{aligned}
& \sum_{l=1}^{N_{x}} \sum_{m=1}^{N_{y}} e^{-i l \mathbf{q}_{s} \cdot \hat{x} d_{x}} e^{-i m \mathbf{q}_{s} \cdot \hat{y} d_{y}} \\
& =e^{-i \mathbf{q}_{s} \cdot\left(\hat{x} d_{x}+\hat{y} d_{y}\right)}\left(\frac{1-e^{-i N_{x} \mathbf{q}_{s} \cdot \hat{x} d_{x}}}{1-e^{-i \mathbf{q}_{s} \cdot \hat{x} d_{x}}}\right)\left(\frac{1-e^{-i N_{y} \mathbf{q}_{s} \cdot \hat{y} d_{y}}}{1-e^{-i \mathbf{q}_{s} \cdot \hat{y} d_{y}}}\right) .
\end{aligned}
$$

These terms are very small unless $\mathbf{q}_{s} \cdot \hat{x} d_{x}=2 n \pi$ and $\mathbf{q}_{s} \cdot \hat{y} d_{y}=$ $2 m \pi$ are integer multiples of $2 \pi$. These two conditions lead to the diffraction equation in two dimensions. For example, writing $\mathbf{k}=k\left(\hat{x} \sin \theta_{I}-\hat{z} \cos \theta_{I}\right)$ and $\mathbf{k}_{s}=k\left(\hat{x} \sin \theta_{S}-\hat{z} \cos \theta_{s}\right)$ with $\theta_{I}$ the incident angle and $\theta_{s}$ the angle of scattering, then $\mathbf{q}_{s} \cdot \hat{x} d_{x}=k d_{x}\left(\sin \theta_{s}-\sin \theta_{I}\right)=2 n \pi$ or on rearranging and substituting $k=2 \pi / \lambda$ we have the condition for strong scattering $\sin \theta_{s}=\sin \theta_{I}+n \lambda / d_{x}$. This is the diffraction equation, which has recently been confused with a generalization of Snell's Law of refraction [41]. For periodicities smaller than the wavelength of light $d \ll \lambda$, the only solution for strong scattering is when 
$n=m=0$ for which $\mathbf{q}_{s} \cdot \hat{x} d_{x}=0$ and $\mathbf{q}_{s} \cdot \hat{y} d_{y}=0$ whereupon $\mathbf{k}_{s}=\mathbf{k}$ and the incident beam is transmitted through the array without deviation. (One can show that a specularly reflected beam also a solution). This demonstrates that, in the weak scattering approximation, a metasurface formed by an array of nanostructures with subwavelength periodicity behaves like a uniform thin film that merely transmits or reflects the incident beam.

\section{FUNDING INFORMATION}

Australian Research Council (DP160100983, FT140100514).

\section{REFERENCES}

1. N. Yu and F. Capasso, "Flat optics with designer metasurfaces," Nat. Mater. 13, 139-150 (2014).

2. A. V. Kildishev, A. Boltasseva, and V. M. Shalaev, "Planar photonics with metasurfaces," Science. 339, 1232009 (2013).

3. A. Pors, M. G. Nielsen, and S. I. Bozhevolnyi, "Analog computing using reflective plasmonic metasurfaces," Nano Lett. 15, 791-797 (2015).

4. A. Silva, F. Monticone, G. Castaldi, V. Galdi, A. Alu, and N. Engheta, "Performing mathematical operations with metamaterials," Science. 343, 160-163 (2014).

5. T. Zhu, Y. Zhou, Y. Lou, H. Ye, Z. R. M. Quu, and S. Fan, "Plasmonic computing of spatial differentiation," Nat. Commun. 8, 15391 (2017).

6. A. Chizari, S. Abdollahramezani, M. Jamali, and J. A. Salehi, "Analog optical computing based on a dielectric meta-reflect array," Opt Lett 41, 3451-3454 (2016).

7. E. Eftekhari, D. E. Gómez, and T. J. Davis, "Measuring subwavelength phase differences with a plasmonic circuit-an example of nanoscale optical signal processing," Opt. Lett. 39, 2994-2997 (2014).

8. T. J. Davis, D. E. Gómez, and A. Roberts, "Plasmonic circuits for manipulating optical information," Nanophotonics. 6, 543-559 (2016).

9. J. Goodman, Introduction to Fourier Optics (Roberts and Company, Greenwood Village, 2005), 3rd ed.

10. L. Maigyte and K. Staliunas, "Spatial filtering with photonic crystals," Appl. Phys. Rev. 2, 011102 (2015).

11. C. Guo, M. Xiao, M. Minkov, Y. Shi, and S. Fan, "Photonic crystal slab Laplace operator for image differentiation," Optica. 5, 251-256 (2018).

12. L. L. Doskolovich, D. A. Bykov, E. A. Bezus, and V. A. Soifer, "Spatial differentiation of optical beams using phase-shifted bragg grating," Opt. Lett. 39, 1278-1281 (2014).

13. D. A. Bykov, L. L. Doskolovich, E. A. Bezus, and V. A. Soifer, "Optical computation of the laplace operator using phase-shifted bragg grating," Opt. Express 22, 25084-25092 (2014).

14. N. V. Golovastikov, D. A. Bykov, L. L. Doskolovich, and E. A. Bezus, "Spatial optical integrator based on phase-shifted bragg gratings," Opt. Commun. 338, 457-460 (2015).

15. C.-L. Dai, Z.-G. Zhao, X.-L. Li, and H.-W. Yang, "Performing derivative and integral operations for optical waves with optical metamaterials," Physics Letters A. 380, 3942-3948 (2016).

16. L. L. Doskolovich, E. A. Bezus, N. V. Golovastikov, D. A. Bykov, and V. A. Soifer, "Planar two-groove optical differentiator in a slab waveguide," Optics Express. 25, 22328-22340 (2017).

17. Y. Wu, Z. Zhuang, L. Deng, Y. Liu, Q. Xue, and Z. Ghassemlooy, "Arbitrary Multi-way Parallel Mathematical Operations Based on Planar Discrete Metamaterials," Plasmonics. 13, 599-607 (2018).

18. W. Wu, W. Jiang, J. Yang, S. Gong, and Y. Ma, "Multilayered analog optical differentiating device: performance analysis on structural parameters," Opt. Lett. 42, 5270-5273 (2017).

19. F. Zangeneh-Nejad, A. Khavasi, and B. Rejaei, "Analog optical computing by half-wavelength slabs," Optics Communications. 407, 338-343 (2018).
20. A. Saba, M. R. Tavakol, P. Karimi-Khoozani, and A. Khavasi, "TwoDimensional Edge Detection by Guided Mode Resonant Metasurface," IEEE Photonics Technology Letters. 30, 853-856 (2018).

21. A. Youssefi, F. Zangeneh-Nejad, S. Abdollahramezani, and A. Khavasi, "Analog computing by brewster effect," Opt. Lett 41, 3467-3470 (2016).

22. B. Vohnsen and D. Valente, "Surface-plasmon-based wavefront sensing," Optica 2, 1024-1027 (2015).

23. Y. Hwang and T. J. Davis, "Optical metasurfaces for subwavelength difference operations," Appl. Phys. Lett. 109, 181101 (2016).

24. Y. Hwang, T. J. Davis, J. Lin, and X.-C. Yuan, "Plasmonic circuit for second-order spatial differentiation at the subwavelength scale," Opt. Express 26, 7368-7375 (2018).

25. T. J. Davis, D. E. Gómez, and F. Eftekhari, "All-optical modulation and switching by a metamaterial of plasmonic circuits," Opt. Lett. 39, 4938-4941 (2014).

26. A. Bykov, L. Doskolovich, A. Morozov, E. Podlipnov, V.V.and Bezus, P. Verma, and V. Soifer, "First-order optical spatial differentiator based on a guided-mode resonant grating," Opt. Express 26, 10997 - 11006 (2018).

27. Z. Dong, J. Si, X. Yu, and X. Deng, "Optical spatial differentiator based on subwavelength high-contrast gratings," Appl. Phys. Lett. 112, 18102 (2018).

28. M. Mansuripur, "Certain computational aspects of vector diffraction problems," J. Opt. Soc. Am. A 6, 786-805 (1989).

29. T. J. Davis and D. E. Gómez, "Colloquium: An algebraic model of localized surface plasmons and their interactions," Rev. Mod. Phys. $\mathbf{8 9}$ 011003 (2017).

30. C.-C. Chen, "Transmission of microwaves through perforated flat plates of finite thickness," IEEE Transactions on Microwwave Theory Tech. 21, 1-6 (1973).

31. A. Roberts and R. C. McPhedran, "Bandpass grids with annular apertures," IEEE Transactions on Antennas Propag. 36, 607-611 (1988).

32. J. D. Jackson, Classical electrodynamics (John Wiley \& Sons Inc, New York, 1975), 2nd ed.

33. L. C. Botten, R. C. McPhedran, and J. M. Lamarre, "Inductive grids in the resonant region - theory and experiment," Int. J. Infrared Millim. Waves 6, 511-575 (1985).

34. R. C. Compton, R. C. McPhedran, G. H. Derrick, and L. C. Botten, "Diffraction properties of a bandpass grid," Infrared Phys. 23, 239-245 (1983).

35. R. C. McPhedran and D. Maystre, "On the theory and solar application of inductive grids," Appl. Phys. 14, 1-20 (1977).

36. R. C. Compton, "Approximation techniques for planar periodic structures," IEEE Transactions on Microw. Theory Tech. 33, 1083-1088 (1985).

37. T. Davis, K. Vernon, and D. Gomez, "A plasmonic "ac wheatstone bridge" circuit for high-sensitivity phase measurement and singlemolecule detection," J. Appl. Phys. 106, 043502 (2009).

38. F. I. Baida, "Enhanced transmission through subwavelength metallic coaxial apertures by excitation of the tem mode," Appl. Phys. B 89, 145-149 (2007).

39. A. Roberts, "Beam transmission through hole arrays," Opt. Express 18, 2528-2533 (2010).

40. N. Marcuvitz, Waveguide Handbook (McGraw-Hill, New York, 1951).

41. N. Yu, P. Genevet, M. Kats, F. Aieta, J. Tetienne, F. Capasso, and Z. Gaburro, "Light propagation with phase discontinuities: Generalized laws of reflection and refraction," Science 334, 333 - 337 (2011). 


\section{University Library}

\section{- M M N E R VA A gateway to Melbourne's research publications}

Minerva Access is the Institutional Repository of The University of Melbourne

Author/s:

Roberts, A;Gomez, DE;Davis, TJ

Title:

Optical image processing with metasurface dark modes

Date:

2018-09-01

Citation:

Roberts, A., Gomez, D. E. \& Davis, T. J. (2018). Optical image processing with metasurface dark modes. JOURNAL OF THE OPTICAL SOCIETY OF AMERICA A-OPTICS IMAGE SCIENCE AND VISION, 35 (9), pp.1575-1584. https://doi.org/10.1364/JOSAA.35.001575.

Persistent Link:

http://hdl.handle.net/11343/243783 